\title{
Dynamic heterogeneity in transition metal oxide cathodes
}

\author{
Karena Chapman \\ Stony Brook University, Stony Brook, United States of America; \\ karena.chapman@stonybrook.edu
}

Lithium-rich transition metal oxide cathodes are of intense current interest as higher capacity alternatives to the stoichiometric layered cathodes currently used in today's automotive applications. These Li-rich cathodes store extra energy through extensive high-voltage oxygen oxidation. The mechanism by which the changes in oxygen redox chemistry is accommodated by the cathode remains actively debated, particularly in terms of the structure changes. How does the change in O chemistry impact the structure and dynamics of the transition metal and lithium cations? Without understanding how oxygen oxidation is accommodated by the cathode structure, and how this is linked to performance limitations, we cannot design strategies to mitigate limitations and displace current automotive electrodes or develop new robust electrode chemistries that access additional O-based redox capacity.

Using operando and complementary ex situ X-ray scattering studies (XRD and SAXS) we explore the dynamic restructuring of transition metal cathodes that occurs during cycling. We identify - for the first time - the formation of nanopores within the cathode during $\mathrm{O}$ oxidation. Upon extended cycling, coarsening of residual pores can be linked to performance degradation

Keywords: operando, cathode, small-angle X-ray scattering, powder diffraction 